\title{
História natural das plantas do Maranhão (Brasil) no século XIX segundo a obra do Frei Francisco de Nossa Senhora dos Prazeres
}

Natural history of the plants of Maranhão (Brazil) in the $19^{\text {th }}$ century according to the works of Friar Francisco de Nossa Senhora dos Prazeres

\author{
Jairo Fernando Pereira Linhares (D) | Maria Ivanilde de Araujo Rodrigues ${ }^{\text {II }}$ (D) Claudio Urbano Bittencourt Pinheiro ${ }^{\text {III }}$ \\ 'Pesquisador independente. São Luís, Maranhão, Brasil \\ "Universidade Estadual do Maranhão. São Luís, Maranhão, Brasil \\ "'Universidade Federal do Maranhão. São Luís, Maranhão, Brasil
}

Resumo: $\bigcirc$ intercâmbio de plantas entre as colônias portuguesas ocorreu ao longo de todo um processo de expansão territorial desde o século XV. O objetivo geral deste artigo foi realizar pesquisa documental em "Poranduba maranhense: relação histórica da província do Maranhão", a fim de resgatar a história natural das plantas do Maranhão no século XIX, de modo a atualizar as informações de acordo com o conhecimento botânico atual, e, mais especificamente, conhecer as espécies, as procedências e os seus respectivos usos. Foram extraídos 174 etnômios, dos quais 88,51\% ( $n=154)$ foram identificados pelo menos em nível de família e 11,49\% ( $n=20)$ não tiveram suas identidades botânicas esclarecidas. Destes, $81 \%(n=141)$ são constituídos por espécies nativas e 19\% $(n=33)$, por exóticas. A alimentação humana obteve a maior citação de uso, 31\% $(n=58)$. Os usos madeireiros e medicinais figuraram na sequência, com respectivos $22 \%(n=41)$ e $15 \%(n=28)$. No códice de plantas, constam tanto espécies voltadas para o comércio, como cana-de-açúcar, algodão, tabaco e café, quanto espécies ligadas aos hábitos alimentares do colonizador português, além de grande número de espécies nativas. De maneira geral, a representação de plantas retrata o padrão de colonização.

Palavras-chave: História natural. Flora do Maranhão. Análise documental.

Abstract: The exchange of plants among the Portuguese colonies occurred throughout the process of Portuguese territorial expansion since the 15th century. The main goal of the present project was to carry out documentary research of the work "Poranduba maranhense: Historical relation of the province of Maranhão" and recover the natural history of plants of Maranhão in the 19th century, so as to update the information according to current botanical knowledge, and more specifically, to identify the species, provenances, and their uses. 174 ethnomies were extracted, of which $88.51 \%(n=154)$ were identified at least at the family level and $11.49 \%(n=20)$ did not have their botanical identities clarified. Of these, $81 \%(n=141)$ consisted of native species and 19\% $(n=33)$ of exotic ones. Human food obtained the highest citation of use, $31 \%(n=58)$. Wood and medicinal uses followed, with respective $22 \%(n=41)$ and $15 \%(n=28)$. In the codex of plants there are commercially important species such as: sugarcane, cotton, tobacco, coffee, as well as species linked to the Portuguese colonizer's eating habits, but also a large number of native species. In general, the representation of plants portrays the pattern of colonization.

Keywords: Natural history. Flora of Maranhão. Documentary analysis.

LINHARES, J. F. P., M. I. A. RODRIGUES \& C. U. B. PINHEIRO, 2019. História natural das plantas do Maranhão (Brasil) no século XIX segundo a obra do Frei Francisco de Nossa Senhora dos Prazeres. Boletim do Museu Paraense Emílio Goeldi. Ciências Naturais 14(2): $209-221$. Autor para correspondência: Jairo Fernando Pereira Linhares. Pesquisador independente (jairoivini29@yahoo.com.br).

Recebido em 23/01/2018

Aprovado em 04/06/2019

Responsabilidade editorial: Fernando da Silva Carvalho Filho
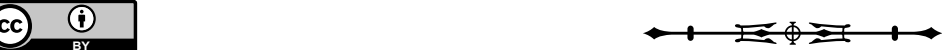


\section{INTRODUÇÃO}

O intercâmbio de plantas entre as colônias ao longo de todo o processo de expansão territorial português, desde o século $X V$, tornou comum o transplante de espécies, especialmente plantas com potencial de exploração agrícola através do cultivo extensivo (Pataca, 2016, p. 90).

Vale ressaltar que, além do cultivo de produtos direcionados ao comércio europeu, como a canade-açúcar, o algodão e o tabaco, os colonizadores portugueses trouxeram hábitos alimentares e, junto com eles, vieram também cultivos de legumes, frutas e verduras já aclimatados, como romãzeira, laranjas, bananas etc. (Gesteira, 2013, p. 34).

No Brasil, o intercâmbio de plantas está tão atrelado à história da colonização que é impossível afirmar a data de chegada de plantas vindas de outras regiões do mundo, muito embora se acredite que tenham vindo com as primeiras caravelas (Kury, 2013, p. 8).

Não obstante, durante o Brasil colônia do século XVII, o monopólio holandês sobre importantes regiões da Ásia que eram produtoras das chamadas 'drogas' (especiarias, tais como condimentos, plantas medicinais e aromáticas etc.), somadas à crise do mercado açucareiro e do tabaco, fez do Maranhão uma alternativa econômica para os portugueses explorarem a riqueza vegetal existente, aliando-se a isso as condições de solo e de clima, as quais eram favoráveis à aclimatação de plantas vindas de diferentes partes do mundo (Cardoso, 2015, p. 118).

Em vista disso, o interesse pelas 'drogas' existentes no Maranhão fez parte de um processo de mudança nas possibilidades de geração de riquezas, iniciado em larga escala entre os séculos XV e XVI, com a implantação de experimentos portugueses que objetivavam a aclimatação de plantas nas ilhas da Madeira, de Cabo Verde e de Açores (Cardoso, 2015, p. 119).

Por outro lado, existia o esforço em cultivar espécies nativas do Maranhão com propriedades semelhantes às atribuídas às drogas vindas do oriente. Após 1670, espécies que eram simplesmente coletadas passaram a ser cultivadas, a exemplo do cacau (Theobroma cacao L. - Malvaceae), cultivado pelo jesuíta Bettendorff, em 1674 (Camargo, 2014, p. 122).

Outros registros sobre a historiografia das plantas no Maranhão durante o período colonial podem ser encontrados em fontes documentais, como a obra escrita no século XVII, intitulada "História da missão dos padres capuchinhos na ilha do Maranhão e suas circumvisinhaças", do missionário francês Frei Claude d'Abbeville (1874), escrita no período em que os franceses invadiram o Maranhão. Após a expulsão dos franceses pelos portugueses, foi enviado ao Maranhão o Frei Cristóvão de Lisboa para catequisar os índios, o qual, durante esse período, escreveu a obra intitulada "História dos animais e árvores do Maranhão" (Lisboa, 1967) (Ávila-Pires, 1992, p. 205).

No século XIX, Frei Francisco de Nossa Senhora dos Prazeres publicou a obra "Poranduba maranhense: relação histórica da província do Maranhão", escrita em 1819 e publicada em 1891, a qual traz uma descrição geral da província do Maranhão e do Pará, até a separação das duas províncias. E justifica a importância de sua obra: "Todos os que têm vivido no Maranhão sabem quão falta é esta província de memórias, e quão custa o alcançar n'ella quaisquer noticias certas" (Prazeres, 1891, p. 8).

No que se refere à importância da obra sobre a história natural do Maranhão, Fr. Francisco de S. Jozé do Convento de São Francisco da Villa-Real, em parecer emitido sobre a referida obra, ressalta:

Em tudo que pertence á historia natural do paiz o autor descreve as couzas com a clareza e exactidão de uma testemunha de vista, pois é sabido que ele viveo muitos anos em muitos sítios, de que fala, onde mesmo delineou e compoz a sua obra (Prazeres, 1891, p. 5-6).

Contudo, apesar do conhecimento acerca da história natural do Maranhão conferido ao Frei Francisco de Nossa 
Senhora dos Prazeres, a descrição das plantas e dos animais foi desprovida de termos técnicos, como o próprio autor esclarece: "No que pertence à história natural omito quazi sempre os termos technicos, e uso de similhanças, para que todos entendam" (Prazeres, 1891, p. 8).

Não obstante, o acesso e a análise das interpretações destas obras vêm propor novas argumentações, trazendo para o presente as evidências que contam sobre o emprego de espécies vegetais da flora nacional e exótica no território brasileiro (Medeiros, 2009, p. 42).

Portanto, a importância do presente artigo consiste no aumento do conhecimento sobre as espécies vegetais presentes no Maranhão no século XIX. O objetivo geral deste artigo foi, portanto, o de realizar pesquisa documental da obra "Poranduba maranhense: relação histórica da província do Maranhão" (Prazeres, 1891) e resgatar a história natural das plantas do Maranhão no século XIX, de modo a atualizar as informações de acordo com o conhecimento botânico atual e, mais especificamente, conhecer as espécies, as procedências e os seus respectivos usos.

\section{MATERIAL E MÉTODOS}

Para coleta dos dados, foi consultada a edição facsímile da obra do Frei Francisco de Nossa Senhora dos Prazeres, intitulada "Poranduba maranhense: relação histórica da província do Maranhão" (Prazeres, 1891). Esta se constituiu na fonte documental primária para elaboração do códice das plantas maranhenses no século XIX. Deste ponto em diante, neste artigo, passa a ser designada como referência direta (RD).

\section{IDENTIDADE E ORIGEM BOTÂNICA}

Para dar início à identificação botânica, os etnômios das plantas encontrados foram transcritos conforme achados na referência direta, mantendo-se a versão paleográfica como eram conhecidas as plantas na época (Medeiros, 2009, p. 60). Como parte dos etnômios da referência direta teve origem na língua Tupi-Guarani, buscou-se o esclarecimento das identidades botânicas por meio de consultas aos trabalhos de Miranda (1942), Barbosa (1951), Edelweiss (1969), Betts (1981) e Bueno (1982).

Complementarmente, foram buscadas informações sobre a identidade botânica através de consultas feitas a dicionários (Medeiros, 2009, p. 60) de língua portuguesa publicados no século XIX e no início do século $X X$, elaborados por Silva (1831a, 1831b) e Figueiredo (1911). Adicionalmente, foram realizadas consultas a "Chambers's encyclopedia, a dictionary of universal knowledge" (Chambers, 1895).

Contudo, o uso de etnômios necessita de um esclarecimento. Se, por um lado, é uma ferramenta indispensável para caracterização das espécies, por outro, pode incidir em erros. $\bigcirc$ emprego dos etnômios pode levar a dois fenômenos, a saber: homonímia (um mesmo nome popular para designar plantas de espécies diferentes) e sinonímia (vários nomes populares pelos quais se denominam uma única espécie) (Vásquez et al., 2014, p. 467).

Outro recurso utilizado para auxiliar na identificação botânica foi a busca às descrições das plantas encontradas nas referências diretas. Como pode ser observado na descrição do jenipapeiro (Genipa americana L. Rubiaceae) encontrada na obra e transcrita a seguir.

\begin{abstract}
Genipapeiro, arvore de folha verde e escura, grossa e assimilhada ao castanheiro. $\bigcirc$ fruto xamado genipapo é da grandeza de maçan, de casca cinzenta e aspéra, polpa tirante a parda e no interior d'estas muitas pevides; é substancial, porém muito quente, especialmente as pevides. Esta arvore despede-se da folha; porém n'ella permanecem os frutos, e só principiam amadurecer, quando ella já esta vestida de nova folha, e já crescidos os genipapos, que tem que ficar para o anno futuro. $\bigcirc$ genipapo maxo não da fruto (Prazeres, 1891, p. 150-151).
\end{abstract}

Outra questão aventada para a elucidação da identidade botânica das espécies foi a extensão territorial inventariada, daí inferimos a possiblidade da existência de corruptelas. A partir disso, compararam- 
se as características das plantas descritas na referência direta com as descrições contidas em manuais de identificação de plantas da atualidade (nomes e características morfológicas que guardem semelhanças) (Linhares et al., 2018, p. 16).

Algumas RD fazem menção à existência de 'castas' e, dependendo da associação feita na obra, podem levar à conotação da ocorrência de espécies semelhantes ou, ainda, à existência de variedade dentro da própria espécie (variedade no sentido botânico). Outra conotação existente é quando se trata de espécie domesticada; aí o termo 'casta' ganha a conotação de variedade, mas, neste caso, tomando o sentido agronômico.

Sendo assim, para efeito da organização dos dados, foram colocadas as 'castas' entre parênteses, ao lado da respectiva referência direta, preservando-se a versão paleográfica; ou, ainda, quando foi explicitada apenas a quantidade de castas existentes, optou-se por fazer o registro, colocado ao lado da respectiva referência direta.

A nomenclatura botânica empregada foi atualizada com base no banco de dados do Missouri Botanical Garden's VAScular Tropicos (VAST) Nomenclatural Database W3 Tropicos (Missouri Botanical Garden, 2006), além do The International Plant Names Index (IPNI, s. d.), bem como em literatura especializada. Para abreviatura dos nomes dos autores das espécies, adotou-se Brumitt \& Powell (1992).

A determinação da origem das plantas deu-se a partir de registros contidos nas próprias RD, pois muitas delas explicitam sua procedência, como pode ser observado no trecho da obra de Prazeres (1891, p. 159): "São-Caetano (africana) [...] Sendo transportada de Guiné, foi plantada junto a uma capela de S. Caetano, e d'elle tomou o nome". Outra forma utilizada foi feita por meio de pesquisa bibliográfica. As RD não identificadas botanicamente, mas com etnômio de origem TupiGuarani, foram consideradas para efeito desse estudo como nativas, uma vez que as plantas exóticas foram introduzidas pelos próprios colonizadores, sendo, portanto, supostamente conhecidas pelo autor.

\section{USOS}

Em relação aos usos atribuídos às referências diretas, somente foram registrados aqueles escritos de forma clara, pois, segundo estudos realizados por Lévi-Strauss (1986, 1989), o aborígene define e classifica tudo aquilo que lhe tenha utilidade, ou então o que possa lhe causar danos. Porém, Berlin (1992) ampliou essa compreensão, quando, em seus estudos, observou forte tendência de classificação baseada em características que vão além da utilidade ou do dano.

\section{RESULTADOS E DISCUSSÃO}

\section{IDENTIDADE E ORIGEM BOTÂNICA}

Foram extraídas 174 referências diretas contidas na obra de Francisco de Nossa Senhora dos Prazeres, das quais $88,51 \%(n=154)$ foram identificados pelo menos no nível de família; do total, 11,49\% $(n=20)$ das plantas ficaram indeterminados, em decorrência de insuficiências nas descrições contidas na fonte documental. Isso se justifica, em grande parte, pela própria explicação dada por Frei Francisco de Nossa Senhora dos Prazeres, que optou por não usar termos técnicos, a fim de permitir melhor compreensão por parte dos leitores, lançando mão de analogias.

As famílias botânicas representadas com maior número de espécies foram: Fabaceae $(n=24)$; Malvaceae $(n=8)$; Euphorbiaceae $(n=7)$; Moraceae e Rubiaceae $(n=6)$; Anacardiaceae, Cucurbitaceae e Poaceae $(n=5)$.

Quanto à origem, 81\% $(n=141)$ das referências diretas são constituídos por espécies nativas e 19\% ( $n=$ 33), por espécies exóticas.

As identidades botânicas das RD, as sinonímias encontradas e as suas respectivas origens foram dispostas no Apêndice 1. 
A quantidade de plantas exóticas introduzidas no Maranhão despertou a atenção de Frei Francisco de Nossa Senhora dos Prazeres (1891, p. 141): "Muitas plantas medicinaes e varias arvores estrangeiras se têm naturalizado no paiz".

A observação feita pelo frei é corroborada pelas práticas adotadas pelo colonizador português. $\bigcirc$ fluxo de plantas entre as diversas regiões coloniais, bem como entre as capitanias brasileiras, foi marca imprescindível para a criação de novas práticas culturais, expressas na culinária, no consumo dos vegetais, em todo o processo de ocupação territorial e na configuração geográfica do império (Pataca, 2016, p. 91).

Em vista disso, além de se observar plantas de origem extra Brasil, pode-se verificar plantas nativas do país, mas provenientes de outras regiões dele, como a jabuticabeira (Myrciaria cauliflora (Mart.) O. Berg Myrtaceae) e a pitanga (Eugenia uniflora L. - Myrtaceae).

\section{USOS}

Predominantemente, as plantas registradas na obra apresentaram alguma função utilitária, porém algumas delas possuíam mais de uma função, como pode ser exemplificado no seguinte trecho:

Carnaúba... tronco muito duro, o qual depois de brunido fica salpicado; e por isso d'elle se fazem lindos bastões. O fruto é negro e todos os viventes o comem. Só com esta planta se pôde fazer uma caza, sem outro ingrediente mais, que cipó (para atar em lugar de pregos) e barro; o tronco dá esteios, barrotes, e ripas; a folha serve para cobrir (como também para outros muitos uzos). Em quanto nova se faz do tronco mais tenro uma espécie de farinha em tempo de fome (Prazeres, 1891, p. 147).

Em vista disso, das 174 referências diretas existentes na obra, 189 apresentavam alguma indicação de uso. A alimentação humana obteve a maior citação, 31\% ( $n=58)$, com destaque para as plantas frutíferas. Os usos madeireiros e medicinais figuraram na sequência, com respectivos $22 \%(n=41)$ e $15 \%(n=28)$.
As referências diretas foram distribuídas em dezessete categorias de uso, como pode ser observado no Apêndice 2.

Por outro lado, algumas plantas, como açoutacavalo, angelim, angelim-de-coco, araribá, árvore-do-pão, bredo, cajurú, figueira, geribéba, não tiverem seus usos revelados por alguma razão. Contudo, algumas podem ter sido catalogadas por simples curiosidade, como pode ser visto no trecho:

\begin{abstract}
Malicia das mulheres, erva espinhosa, de folha assimilhada à da lentilha, porém mais muida. Tanto que se lhe toca em algum ramo, logo os olhos d'este se murcham, e cada uma folha se encosta a sua oposta; mas passado algum espaço, recobra a planta o seo antigo estado. Desde o pôr até ao nascer do sol sucede em toda esta planta a mesma murchidão. Alguns autores xamam-lhe mimoza ou sensitiva (Prazeres, 1891, p. 153)
\end{abstract}

Outro aspecto a ser observado na obra é o emprego de sinonímias que, em alguns casos, resultou no tratamento de espécies distintas como se fosse uma única espécie, como pode ser observado no caso do cipó-de-cobra (Cissampelos glaberrima A. St. -Hil. - Menispermaceae) e da raiz-de-cobra (Polygonum bistorta L. - Polygonaceae) (Figuras 1A e 1B). O cipó-de-cobra é uma espécie nativa, enquanto que a raiz-de-cobra, de origem europeia, já era conhecida quanto ao seu emprego no tratamento contra picada de cobra (Chambers's, 1895). Chegando a terras brasileiras, o colonizador português deparou-se com outra planta, o cipó-de-cobra, a que os índios atribuíam a mesma propriedade; em razão disso, elas foram agrupadas como pertencendo a uma única espécie.

\section{CONCLUSÃO}

No códice de plantas, constam tanto espécies voltadas ao comércio - como cana-de-açúcar, algodão, tabaco, café quanto as ligadas aos hábitos alimentares do colonizador português, além de um grande número de espécies nativas. De maneira geral, a representação de plantas retrata o padrão de colonização. 


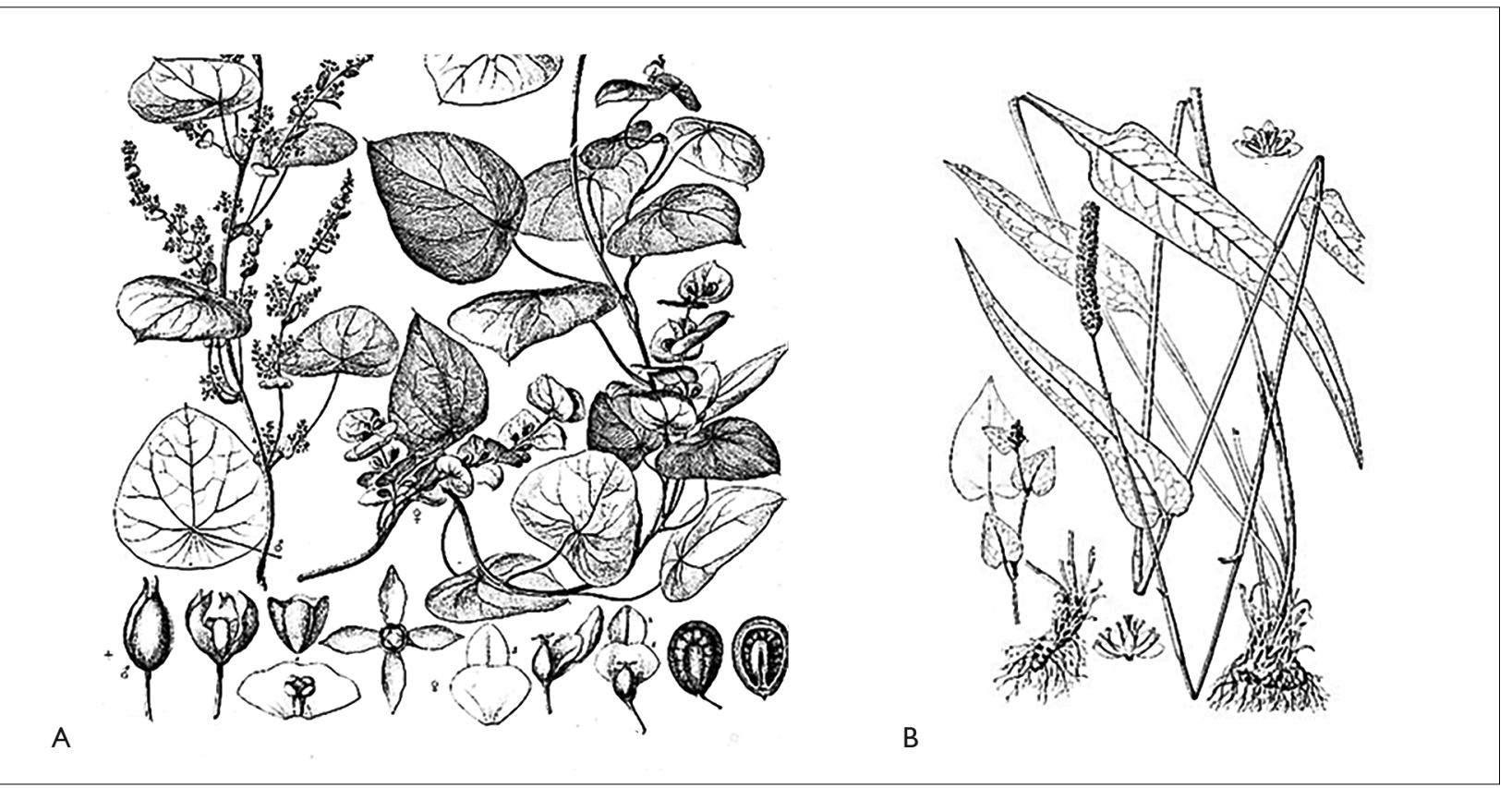

Figura 1. A) Cipó-de-cobra (Cissampelos glaberrima A. St. -Hill. - Menispermaceae); B) raiz-de-cobra (Polygonum bistorta L. - Polygonaceae). Fontes: Plantillustrations.org (s. d.) (A) e Missouri Botanical Garden (s. d.) (B).

\section{REFERÊNCIAS}

ÁVILA-PIRES, F. D., 1992. Mamíferos descritos na Poranduba maranhense de Frei Francisco dos Prazeres. Revista Brasileira de Zoologia 9(3-4): 203-213. DOI: http://dx.doi.org/10.1590/S010181751992000200006.

BARBOSA, L., 1951. Pequeno vocabulário Tupi-Português: 1-202. Livraria São José, Rio de Janeiro.

BERLIN, B., 1992. Ethnobiological classification: principles of categorization of plants and animals in traditional societies: 1-335. Princeton University Press, Princeton.

BETTS, L. V., 1981. Dicionário Paritintin: 1-231. Sociedade Internacional de Linguística, Departamento de Programas Linguísticos, Cuiabá.

BRUMITT, R. K. \& C. E. POWELL (Ed.), 1992. Authors of plant names: a list of authors of scientific names of plants, with recommended standard forms of their names, including abbreviations. Royal Botanic Garden Kew, London.

BUENO, F. S., 1982. Vocabulário tupi-guarani português: 1-672. Brasil Livros Editor e Distribuidor Ltda., São Paulo.

CAMARGO, M. T. L. A., 2014. As plantas medicinais e o sagrado: a etnofarmacobotânica em uma revisão historiográfica da medicina popular no Brasil: 1. ed.: 1-280. Ícone, São Paulo.
CARDOSO, A., 2015. Species in Portuguese Amazon region: vegetable retail and Atlantic trade in the end of the Spanish Monarchy. Tempo 21(37): 1-18. DOI: https://doi.org/10.1590/ TEM-1980-542X2015v213701.

CHAMBERS, W., 1895. Chambers's encyclopedia a dictionary of universal knowledge: v. II: 1-828. Beaugency to cataract. William \& Robert Chambers. Limited/J.B. Lippincott Company, London and Edinburgh/Philadelphia.

D'ABBEVILLE, C., 1874. História da missão dos padres capuchinhos na ilha do Maranhão e suas circumvisinhaças. Typ. do Frias, São Luiz.

EDELWEISS, F. G., 1969. Estudos Tupis e Tupis-Guaranis: confrontos e revisões: 1-299. Livraria Brasiliana Editora, Rio de Janeiro.

FIGUEIREDO, C., 1911. Novo dicionário da língua portuguesa: v. I: 1-1010. Sociedade Editora Arthur Brandão, Lisboa.

GESTEIRA, H. M., 2013. A América portuguesa e a circulação de plantas, séculos XVI-XVIII. In: L. KURY (Org.): Usos e circulação de plantas no Brasil - séculos XVI-XIX: 1. ed.: 1-324. Andrea Jakobson Estúdio Editorial Ltda., Rio de Janeiro.

KURY, L., 2013. Apresentação. In: L. KURY (Org.): Usos e circulação de plantas no Brasil - séculos XVI-XIX: 1. ed.: 8. Andrea Jakobson Estúdio Editorial Ltda., Rio de Janeiro.

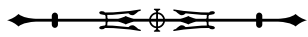


LÉVI-STRAUSS, C., 1986. O uso de plantas silvestres da América do Sul tropical. In: D. RIBEIRO (Ed.): Suma etnológica brasileira: Etnobiologia: v. 1: 27-46. Vozes/FINEP, Petrópolis.

LÉVI-STRAUSS, C., 1989. A ciência do concreto. In: C. LÉVISTRAUSS. O pensamento selvagem: 15-49. Editora Papirus, Campinas, SP.

LINHARES, J. F. P., M. I. A. RODRIGUES, P. F. FREITAS \& C. U. B. PINHEIRO, 2018. Etnobotânica histórica das plantas do Maranhão no século XVII baseada na obra de Cristóvão de Lisboa. Biota Amazônia 8(3): 15-18. DOI: http://dx.doi.org/10.18561/21795746/biotaamazonia.v8n3p15-18.

LISBOA, F. C., 1967. História dos animais e árvores do Maranhão: 1-448. Arquivo Histórico Ultramarino/Centro de Estudos Históricos Ultramarinos, Lisboa.

MEDEIROS, M. F. T., 2009. Etnobotânica histórica: princípios e procedimentos: 1-84. NUPEEA/Sociedade Brasileira de Etnobiologia e Etnoecologia (Série Estudos e Debates), Recife.

MIRANDA, V. C., 1942. Estudos sobre o Nhêengatú. In: R. GARCIA (Ed.): Exotismos franceses originários da Língua Tupi. Anais da Biblioteca Nacional do Rio de Janeiro 64: 129-175.

MISSOURI BOTANICAL GARDEN, 2006. Disponível em: http:// www1.biologie.uni-hamburg.de/b-online/ibc99/mobot. Acesso em: 20 julho 2019.

MISSOURI BOTANICAL GARDEN, [s. d.]. Tropicos.org. Disponível em: http://www.tropicos.org/Image/19782. Acesso em: 20 julho 2019.
PATACA, E. M., 2016. Coleta, transporte e aclimatação de plantas no Império Luso-Brasileiro (1777-1822). Museologia \& Interdisciplinaridade 5(9): 88-108. DOI: https://doi.org/10.26512/ museologia.v5i9.17247.

PLANTILLUSTRATIONS.ORG, [s. d.]. Cissampelos glaberrima A.St.-Hil. Disponível em: http://www.plantillustrations.org/ illustration.php?id_illustration $=12756 \&$ SID $=0 \&$ mobile $=0 \&$ co de_category_taxon=1\&size $=1$. Acesso em: 20 julho 2019 .

PRAZERES, F. F. N. S, 1891. Poranduba maranhense: relação histórica da província do Maranhão. Revista Trimensal do Instituto Histórico e Geographico Brazileiro 54(pt. 1): [4]-277. Disponível em: http://biblio.etnolinguistica.org/prazeres_1891_poranduba. Acesso em: 14 outubro 2017.

SILVA, A. M., 1831a. Dicionário da língua portuguesa: Tomo I: A-E: 1-812. [S. n.], Lisboa.

SILVA, A. M., 1831b. Dicionário da língua portuguesa: Tomo II: F-Z: 1-906. [S. n.], Lisboa.

THE INTERNATIONAL PLANT NAMES INDEX (IPNI), [s. d.]. Disponível em: http://www.ipni.org/ipni/plantnamesresearchpage. Acesso em: 20 julho 2019.

VÁSQUEZ, S. P. F., M. S. MENDONÇA \& S. N. NODA, 2014. Etnobotânica de plantas medicinais em comunidades ribeirinhas do município de Manacapuru, Amazonas, Brasil. Acta Amazônica 44(4): 457-472. DOI: http://dx.doi.org/10.1590/18094392201400423. 
Apêndice 1. Referências diretas (RD) citadas na obra de Francisco de Nossa Senhora dos Prazeres das plantas maranhenses no século XIX, com as respectivas famílias botânicas, nomes científicos e origem das plantas.

\begin{tabular}{|c|c|c|c|c|}
\hline$N^{\circ}$ & Família botânica & Nome científico & RD/castas & Origem \\
\hline 1 & Amaranthaceae & Amaranthus spinosus L. 1753 & Bredo & Nativa \\
\hline 2 & Amaranthaceae & Dysphania ambrosioides (L.) Mosyakin \& Clemants 2002 & Mastrús & Exótica \\
\hline 3 & Anacardiaceae & Anacardium occidentale L. 1753 & Cajueiro (vermelho e amarelo) & Nativa \\
\hline 4 & Anacardiaceae & Mangifera indica L. 1753 & Mangueira (duas castas) & Exótica \\
\hline 5 & Anacardiaceae & Myracrodruon urundeuva Allemão 1862 & Aroeira & Nativa \\
\hline 6 & Anacardiaceae & Spondias mombin L. 1753 & Cajazeiras & Nativa \\
\hline 7 & Anacardiaceae & Tapiripa guianensis Aubl. 1775 & Tamanca & Nativa \\
\hline 8 & Annonaceae & Annona coriacea Mart. 1841 & Araticum & Nativa \\
\hline 9 & Annonaceae & Duguetia furfuracea (A.St.-Hil.) Saff. 1914 & Atéira & Nativa \\
\hline 10 & Annonaceae & Annona squamosa L. 1753 & Condeceira & Nativa \\
\hline 11 & Apocynaceae & Hancornia speciosa Gomes 1803 & Mangabeira (mangabeira brava) & Nativa \\
\hline 12 & Apocynaceae & Parahancornia amapa (Huber) Ducke 1922 & Mapá & Nativa \\
\hline 13 & Apocynaceae & Aspidosperma nitidum Benth. ex Müll. Arg. 1860 & Páo de remo & Nativa \\
\hline 14 & Araceae & Colocasia esculenta (L.) Schott 1832 & Inhâme & Nativa \\
\hline 15 & Arecaceae & Acrocomia aculeata (Jacq.) Lodd. ex Mart. 1845 & Mucajúba & Nativa \\
\hline 16 & Arecaceae & Astrocaryum vulgare Mart. 1824 & Tucum (linho rijo) & Nativa \\
\hline 17 & Arecaceae & Bactris brongniartii Mart. 1844 & Marajá & Nativa \\
\hline 18 & Arecaceae & Cocos nucifera L. 1753 & Coqueiro & Exótica \\
\hline 19 & Arecaceae & Copernicia prunifera (Mill.) H.E. Moore 1963 & Carnaúba & Nativa \\
\hline 20 & Arecaceae & Desmoncus anomalus Bartlett 1935 & Titara & Nativa \\
\hline 21 & Arecaceae & Euterpe oleracea Mart. 1824 & Juçara & Nativa \\
\hline 22 & Arecaceae & Mauritia flexuosa L. f. 1781 & Murutim & Nativa \\
\hline 23 & Arecaceae & Maximiliana maripa (Aubl.) Drude 1881 & Anajá & Nativa \\
\hline 24 & Arecaceae & Oenocarpus bacaba Mart. 1823 & Bacába & Nativa \\
\hline 25 & Arecaceae & Orbignya phalerata Mart. 1884 & Palmeira, pindobeira & Nativa \\
\hline 26 & Arecaceae & Syagrus cocoides Mart. 1881 & Iriri & Nativa \\
\hline 27 & Arecaceae & Attalea maripa (Aubl.) Mart. & Perinan & Nativa \\
\hline 28 & Asteraceae & Eremanthus Less. 1829 & Candeia & Nativa \\
\hline 29 & Bignoniaceae & Crescentia cujete L. 1753 & Cuieira & Nativa \\
\hline 30 & Bignoniaceae & Handroanthus Mattos 1970 & Cedro & Nativa \\
\hline 31 & Bignoniaceae & Tabebuia vellosoi Tol. 1952 & Páo d'arco (existem quatro castas) & Nativa \\
\hline 32 & Bixaceae & Bixa orellana L. 1753 & Urucú & Nativa \\
\hline 33 & Bromeliaceae & Ananas Mill. 1754 & Ananozeiro; ananáz & Nativa \\
\hline 34 & Bromeliaceae & Neoglaziovia variegata (Arruda) Mez 1923 & Croatá & Nativa \\
\hline 35 & Burseraceae & Protium heptaphyllum (Aubl.) Marchand 1873 & Almécega, páo de breu & Nativa \\
\hline 36 & Caricaceae & Carica papaya L. 1753 & Mamoeiro (existem três castas) & Nativa \\
\hline
\end{tabular}

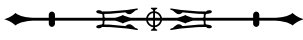


Apêndice 1.

\begin{tabular}{|c|c|c|c|c|}
\hline $\mathrm{N}^{\circ}$ & Família botânica & Nome científico & $\mathrm{RD} /$ castas & Origem \\
\hline 37 & Cariocaraceae & Caryocar brasiliense Cambess. 1825 & Pequizeiro (existem duas castas) & Nativa \\
\hline 38 & Cariocaraceae & Caryocar villosum (Aubl.) Pers. 1806 & Pequirana & Nativa \\
\hline 39 & Clusiaceae & Platonia insignis Mart. 1829 & $\begin{array}{l}\text { Bacurizeiro (branco, } \\
\text { vermelho, amarelo) }\end{array}$ & Nativa \\
\hline 40 & Combretaceae & Terminalia brasiliensis Spreng. 1825 & Catinga de porco & Nativa \\
\hline 41 & Combretaceae & Laguncularia racemosa (L.) C.F. Gaertn 1807 & Mangue branco & Nativa \\
\hline 42 & Convolvulaceae & Ipomoea batatas (L.) Lam. 1793 & Batata & Nativa \\
\hline 43 & Chrysobalanaceae & Chrysobalanus icaco L. 1753 & Cajurú & Nativa \\
\hline 44 & Clusiaceae & Symphonia globulifera L. f. 1782 & Guandi & Nativa \\
\hline 45 & Cucurbitaceae & Citrullus lanatus (Thunb.) Matsum. \& Nakai 1916 & Melancia & Exótica \\
\hline 46 & Cucurbitaceae & Cucumis melo L. 1753 & Melão & Exótica \\
\hline 47 & Cucurbitaceae & Luffa operculata (L.) Cogn. 1878 & Buxa de paulista & Nativa \\
\hline 48 & Cucurbitaceae & Momordica charantia L. 1753 & São-caetano & Exótica \\
\hline 49 & Cucurbitaceae & Sicana odorifera (Vell.) Naudin 1862 & Croá; melão de cabôclo & Nativa \\
\hline 50 & Dioscoreaceae & Dioscorea alata L. 1753 & $\begin{array}{c}\text { Cará (duas variedades não } \\
\text { especificadas) }\end{array}$ & Nativa \\
\hline 51 & Dilleniaceae & Curatella americana L. 1753 & Sambahiba & Nativa \\
\hline 52 & Euphorbiaceae & Euphorbia hirta L. 1753 & Capéva, erva de Santa Luzia & Nativa \\
\hline 53 & Euphorbiaceae & Croton cajucara Benth. 1854 & Sacáca & Nativa \\
\hline 54 & Euphorbiaceae & Croton urucurana Baill. 1864 & Urucurâna & Nativa \\
\hline 55 & Euphorbiaceae & Jatropha gossypiifolia L. 1753 & Pião; pinhão & Exótica \\
\hline 56 & Euphorbiaceae & Manihot esculenta Crantz 1766 & Mandioca, maniva; aipim; macaxeira & Nativa \\
\hline 57 & Euphorbiaceae & Ricinus communis L. 1753 & Carrapateiro, mamona & Exótica \\
\hline 58 & Euphorbiaceae & Cnidoscolus adenochlamys Fern. Casas 2004 & Urtiga de rato & Nativa \\
\hline 59 & Fabaceae & Anadenanthera colubrina (Vell.) Brenan 1955 & Angico, paricá & Nativa \\
\hline 60 & Fabaceae & Andira fraxinifolia Benth. 1837 & Angelim & Nativa \\
\hline 61 & Fabaceae & Não identificada & Angelim de côco & Nativa \\
\hline 62 & Fabaceae & Arachys hypogea L. 1753 & Mandubi & Nativa \\
\hline 63 & Fabaceae & Não identificada & Cotiúba & Nativa \\
\hline 64 & Fabaceae & Glycyrrhiza glabra L. 1753 & Alcaçús & Exótica \\
\hline 65 & Fabaceae & Bowdichia virgilioides Kunt. 1823 & Sucupira & Nativa \\
\hline 66 & Fabaceae & Caesalpinia ferrea Mart. ex Tul. 1844 & Geruparibóra, páo-ferro & Nativa \\
\hline 67 & Fabaceae & Cajanus cajan (L.) Huth. 1893 & Guandú & Exótica \\
\hline 68 & Fabaceae & Copaiva officinalis Jacq. 1760 & Copaúba, copahiba & Nativa \\
\hline 69 & Fabaceae & Dalbergia nigra (Vell.) Allemão ex Benth. 1860 & Páo-santo & Nativa \\
\hline 70 & Fabaceae & Dipteryx odorata (Aubl.) Forssyth f. 1794 & Cumarú & Nativa \\
\hline 71 & Fabaceae & Hymenaea courbaril L. 1753 & Jatobá, jutahi (grande e mirim) & Nativa \\
\hline 72 & Fabaceae & Indigofera L. 1753 & Anil & Exótica \\
\hline
\end{tabular}


Apêndice 1.

\begin{tabular}{|c|c|c|c|c|}
\hline$N^{0}$ & Família botânica & Nome científico & $\mathrm{RD} /$ castas & Orioem \\
\hline 73 & Fabaceae & Inga Mill 1754 & Ingá & Nativa \\
\hline 74 & Fabaceae & Machaerium aculeatum Raddi. 1820 & Campéxe & Nativa \\
\hline 75 & Fabaceae & Phaseolus vulgaris L. 1753 & Feijão & Exótica \\
\hline 76 & Fabaceae & Mimosa pudica L. 1753 & $\begin{array}{c}\text { Malicia das mulheres, mimoza; } \\
\text { sensitiva }\end{array}$ & Nativa \\
\hline 77 & Fabaceae & Caesalpinia bracteosa Tul. 1884 & Páo de rato & Nativa \\
\hline 78 & Fabaceae & Tamarindus indica L. 1753 & Tamarindo & Exótica \\
\hline 79 & $\begin{array}{c}\text { Fabaceae } \\
\text { Euphorbiaceae } \\
\text { Asteraceae }\end{array}$ & $\begin{array}{c}\text { Tephrosia Pers. } 1807 \\
\text { Euphorbia cotinifolia L. } 1753 \\
\text { Clibadium F. Allam. ex L. } 1771\end{array}$ & Timbó & Nativa \\
\hline 80 & Fabaceae & Peltogyne Vogel 1837 & Páo-rôxo & Nativa \\
\hline 81 & Fabaceae & Enterolobium timbouva Mart. 1837 & Timbaúba & Nativa \\
\hline 82 & Fabaceae & Centrolobium tomentosum Guill. ex Benth. 1840 & Araribá & Nativa \\
\hline 83 & Geraniaceae & Geranium L. 1753 & Almiscar & Exótica \\
\hline 84 & Hypericaceae & Vismia brasiliensis Choisy 1821 & Páo de envira & Nativa \\
\hline 85 & Hypericaceae & Vismia Vand. 1788 & Páo de lacre & Nativa \\
\hline 86 & Lauraceae & Persea americana Mill. 1768 & Abacateiro & Exótica \\
\hline 87 & Lauraceae & Laurus nobilis L. 1753 & Louro & Nativa \\
\hline 88 & Lecythidaceae & Lecythis pisonis Cambess. 1829 & Sapucaia & Nativa \\
\hline 89 & Lecythidaceae & Lecythis Loefl. 1758 & Amejuba (branca e amarela) & Nativa \\
\hline 90 & Lecythidaceae & Cariniana legalis (Mart.) Kuntze 1898 & Páo de estopa; táuari & Nativa \\
\hline 91 & Lecythidaceae & Lecythis Loefl. 1758 & Inhaúba (existem duas castas) & Nativa \\
\hline 92 & Lecythidaceae & Cariniana micrantha Ducke 1930 & Tauarí1 & Nativa \\
\hline 93 & Lythraceae & Punica granatum L. 1753 & Romeira & Exótica \\
\hline 94 & Loranthaceae & $\begin{array}{c}\text { Struthanthus Mart. } 1830 . \\
\text { Phthirusa pyrifolia (Kunth) Eichler } 1868 \\
\text { Psittacanthus Mart. } 1830\end{array}$ & Erva de passarinho, temtém & Nativa \\
\hline 95 & Malpighiaceae & Byrsonima Rich. ex Kunth. 1821 & Muruci & Nativa \\
\hline 96 & Malvaceae & Hibiscus sabdariffa L. 1753 & Vinagreira & Exótica \\
\hline 97 & Malvaceae & Luehea divaricata Mart. 1826 & Açouta-cavalo & Nativa \\
\hline 98 & Malvaceae & Ceiba pentandra L. (Gaertn.) 1791 & Barriguda, sumaúma & Nativa \\
\hline 99 & Malvaceae & Gossypium arboreum L. 1753 & Algodoeiro & Nativa \\
\hline 100 & Malvaceae & Sterculia chicha A. St.-Hil. ex Turpin 1817 & Axixá & Nativa \\
\hline 101 & Malvaceae & Theobroma cacao L. 1753 & Cacauzeiro & Nativa \\
\hline 102 & Malvaceae & Malva sylvestris L. 1753 & Malva (preta, branca, brava) & Nativa \\
\hline 103 & Malvaceae & Abelmoschus esculentus (L.) Moench. 1794 & $\begin{array}{c}\text { Quiabeiro, kingobô (existe uma } \\
\text { casta) }\end{array}$ & Exótica \\
\hline 104 & Melastomataceae & Mouriri Aubl. 1775 & Criurí & Nativa \\
\hline 105 & Meliaceae & Carapa guianensis Aubl. 1775 & Andirobeira & Nativa \\
\hline
\end{tabular}

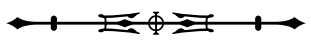


Apêndice 1.

\begin{tabular}{|c|c|c|c|c|}
\hline$N^{\circ}$ & Família botânica & Nome científico & $\mathrm{RD} /$ castas & Origem \\
\hline 106 & Menispermaceae & Tiliacora chrysobotrya, Welw. ex Ficalho 1884 & Bútua & Nativa \\
\hline 107 & Moraceae & Ficus L. 1753 & Gameleira & Nativa \\
\hline 108 & Moraceae & Ficus carica L. 1753 & Figueira & Exótica \\
\hline 109 & Moraceae & Artocarpus heterophyllus Lam. 1789 & Jaqueira & Exótica \\
\hline 110 & Moraceae & Bagassa guianensis Aubl. 1775 & Tatajuba (existem 4 castas) & Nativa \\
\hline 111 & Moraceae & Dorstenia brasiliensis Lam. 1786 & Contraerva & Nativa \\
\hline 112 & Moraceae & Artocarpus altilis (Parkinson) Fosberg 1941 & Arvore do pão & Exótica \\
\hline 113 & Musaceae & Musa $\times$ paradisiaca L. 1753 & $\begin{array}{l}\text { Bananeira, pacoveira (curta, } \\
\text { comprida, rabixa, tangerina). }\end{array}$ & Exótica \\
\hline 114 & Myrtaceae & Campomanesia xanthocarpa Mart. ex O. Berg. 1857 & Guabirabeira & Nativa \\
\hline 115 & Myrtaceae & Eugenia uniflora L. 1753 & Pitanga & Nativa \\
\hline 116 & Myrtaceae & Myrciaria cauliflora (Mart.) O.Berg. 1856 & Jaboticabeira & Nativa \\
\hline 117 & Myrtaceae & Psidium guajava L. 1753 & Goiabeira & Nativa \\
\hline 118 & Myrtaceae & Psidium kennedyanum Morong. 1893 & Araçá & Nativa \\
\hline 119 & Myrtaceae & Syzygium jambos (L.) Alston 1931 & Jambeiro & Exótica \\
\hline 120 & Myrtaceae & Myrcianthes pungens (O. Berg) D. Legrand 1968 & Guabijú & Nativa \\
\hline 121 & Opiliaceae & Agonandra brasiliensis Miers ex Benth. \& Hook. f. 1862 & Gepió & Nativa \\
\hline 122 & Orchidaceae & Vanilla Mill. 1754 & Baunilha & Nativa \\
\hline 123 & Passifloraceae & Passiflora L. 1753 & Maracujá (mirim e assú) & Nativa \\
\hline 124 & Poaceae & Bambusa vulgaris Schrad. ex J.C. Wendl. 1808 & Taboca ou cana & Nativa \\
\hline 125 & Poaceae & Bambusa guadua Bonpl. 1808 & Tocoára & Nativa \\
\hline 126 & Poaceae & Oryza sativa L. 1753 & Arroz & Exótica \\
\hline 127 & Poaceae & Saccharum officinarum L. 1753 & Cana d' assucar & Exótica \\
\hline 128 & Poaceae & Zea mays L. 1753 & Milho, milhão & Exótica \\
\hline 129 & Polygonaceae & Polygonum punctatum Elliot 1821 & Erva de bixo & Nativa \\
\hline 130 & Polygonaceae & Polygonum bistorta L. 1753 & Raiz de cobra & Exótica \\
\hline 131 & Polygonaceae & Triplaris Loefl. 1758 & Taquipé & Nativa \\
\hline 132 & Pontederiaceae & Eichhornia crassipes (Mart.) Solms. 1883 & Mururi & Nativa \\
\hline 133 & Rhizophoraceae & Rhizophora mangle L. 1753 & Mangue vermelho & Nativa \\
\hline 134 & Rubiaceae & Palicourea marcgravii A. St.-Hil. 1824 & Erva de rato & Nativa \\
\hline 135 & Rubiaceae & Cinchona L. 1753 & Quina & Nativa \\
\hline 136 & Rubiaceae & Coffea arabica L. 1753 & Cafezeiro & Exótica \\
\hline 137 & Rubiaceae & Genipa americana L. 1753 & Genipapeiro & Nativa \\
\hline 138 & Rubiaceae & Psychotria ipecacuanha (Brot.) Standl. 1916 & $\begin{array}{l}\text { Ipecacuanha, pacacônha } \\
\text { (escura, parda, branca). }\end{array}$ & Nativa \\
\hline 139 & Rubiaceae & $\begin{array}{c}\text { Calycophyllum spruceanum (Benth.) } \\
\text { Hook. f. ex K. Schum. } 1889\end{array}$ & Páo-mamaluco & Nativa \\
\hline 140 & Rutaceae & Citrus $\times$ aurantium L. 1753 & Limoeiro & Exótica \\
\hline
\end{tabular}


Apêndice 1.

\begin{tabular}{|c|c|c|c|c|}
\hline$N^{\circ}$ & Família botânica & Nome científico & $\mathrm{RD} /$ castas & Origem \\
\hline 141 & Rutaceae & Citrus sinensis (L.) Osbeck. 1765 & Laranjeira & Exótica \\
\hline 142 & Sapindaceae & Talisia esculenta (A. St.-Hill.) Radlk. 1878 & Pitombeira & Nativa \\
\hline 143 & Sapindaceae & Sapindus saponaria L. 1753 & Saboneteiro & Nativa \\
\hline 144 & Sapindaceae & Paullinia cururu L. 1753 & Cururu & Nativa \\
\hline 145 & Sapotaceae & Manilkara huberi (Ducke) A. Cheval. 1932 & Maceranduba (mirim, assú) & Nativa \\
\hline 146 & Sapotaceae & Pouteria adolfi-friedericii (Engl.) A. Meeuse 1960 & Teturubazeiro & Nativa \\
\hline 147 & Simaroubaceae & Simarouba amara Aubl.1775 & Paparaúba (existem duas castas) & Nativa \\
\hline 148 & Solanaceae & Capsicum frutescens L. 1753 & $\begin{array}{c}\text { Pimentas (malagueta e } \\
\text { várias outras castas) }\end{array}$ & Nativa \\
\hline 149 & Solanaceae & Nicotiana tabacum L. 1753 & Fumo, tabaco & Exótica \\
\hline 150 & Talinaceae & Talinum triangulare (Jacq.) Willd. 1799 & João-gomes & Nativa \\
\hline 151 & Tiliaceae & Apeiba tibourbou Aubl. 1775 & Pente de macaco & Nativa \\
\hline 152 & Verbenaceae & Lantana camara L. 1753 & Erva de xumbo & Nativa \\
\hline 153 & Vitaceae & Vitis vinifera L. 1753 & Parreira, videira & Exótica \\
\hline 154 & Zingiberaceae & Zingiber officinale Roscoe 1807 & Gengibre (branco e amarelo) & Exótica \\
\hline 155 & Indeterminada & Não identificada & Bracutiára & Nativa \\
\hline 156 & Indeterminada & Não identificada & Cararoúba & Nativa \\
\hline 157 & Indeterminada & Não identificada & Cipó & Nativa \\
\hline 158 & Indeterminada & Não identificada & Condurú & Nativa \\
\hline 159 & Indeterminada & Não identificada & Geribéba & Nativa \\
\hline 160 & Indeterminada & Não identificada & Gororóba & Nativa \\
\hline 161 & Indeterminada & Não identificada & Gramo da terra & Nativa \\
\hline 162 & Indeterminada & Não identificada & Guarapirarga & Nativa \\
\hline 163 & Indeterminada & Não identificada & Imburagiá & Nativa \\
\hline 164 & Indeterminada & Não identificada & Imburaité & Nativa \\
\hline 165 & Indeterminada & Não identificada & Jacaré-catinga & Nativa \\
\hline 166 & Indeterminada & Não identificada & Jaracatutiba & Nativa \\
\hline 167 & Indeterminada & Não identificada & Juredá & Nativa \\
\hline 168 & Indeterminada & Não identificada & Merim & Nativa \\
\hline 169 & Indeterminada & Não identificada & Moconambi & Nativa \\
\hline 170 & Indeterminada & Não identificada & Parúra & Nativa \\
\hline 171 & Indeterminada & Não identificada & Pequipocúba & Nativa \\
\hline 172 & Indeterminada & Não identificada & Sapupira & Nativa \\
\hline 173 & Indeterminada & Não identificada & Sicantan & Nativa \\
\hline 174 & Indeterminada & Não identificada & Violete & Nativa \\
\hline \multicolumn{3}{|r|}{ Total } & 174 referências diretas & $\begin{array}{l}141 \text { nativas } \\
33 \text { exóticas }\end{array}$ \\
\hline
\end{tabular}


Apêndice 2. Referências diretas/etnômios citados na obra de Francisco de Nossa Senhora dos Prazeres acerca das plantas maranhenses no século XIX, com seus respectivos usos: 1 = alimentação humana; $2=$ madeira; $3=$ medicinal; $4=$ utensílios; $5=$ fibra; $6=$ tóxica; $7=$ corante; 8 = construção; 9 = óleo/azeite; 10 = bebida; $11=$ saponáceas; $12=$ fumo; $13=$ lenha; $14=$ incenso; $15=$ ligante; $16=$ cera; e 17 = aromática. Legendas: RD = referências diretas (grafias de acordo com análise histórica); Qtde = quantidade.

\begin{tabular}{|c|c|c|c|c|}
\hline$N^{\circ}$ & Uso & RD/Etnômio & Qtde & $\%$ \\
\hline 1 & $\begin{array}{l}\text { Alimentação } \\
\text { humana }\end{array}$ & $\begin{array}{c}\text { Cajueiro; mangueira; cajazeiras; araticum; ateira; condeceira; mangabeira; inhame; } \\
\text { carnaúba; juçara; anajá; bacába; pindobeira; ananozeiro; mamoeiro; pequizeiro; } \\
\text { bacurizeiro; batata; melancia; melão; melão de caboclo; cará; mandioca, macaxeira; } \\
\text { mandubi; guandú; jatobá; ingá; feijão; tamarindo; abacateiro; vinagreira; cacauzeiro; } \\
\text { kiabeiro; criurí; jaqueira; bananeira; guabirabeira; pitanga; jaboticabeira; goiabeira; } \\
\text { araçá; jambeiro; baunilha; maracujá; arroz; cana d'assucar; milho; genipapeiro; } \\
\text { limoeiro; laranjeira; pitombeira; pimentas; joão-gomes; videira; jacaré-catinga; } \\
\text { jaracatutiba; sapucaia }\end{array}$ & 58 & 31 \\
\hline 2 & Madeira & $\begin{array}{l}\text { Cajueiro; aroeira; tamanca; mapá; candeia; cedro; angico; páo d’arco; pequirana; } \\
\text { bacurizeiro; guandi; cotiúba; sucupira; geruparibóra; páo-santo; timbaúba; louro; } \\
\text { amejuba; axixá; tatajuba; guabijú; gepió; páo-mamaluco; maceranduba; bracutiára; } \\
\text { cararoúba; condurú; gororóba; guarapirarga; imburagiá; juredá; merim; parúra; } \\
\text { pequipocúba; sapupira; sicantan; cumaru; cururú; imburagiá; inhaúba; jatobá }\end{array}$ & 41 & 22 \\
\hline 3 & Medicinal & $\begin{array}{l}\text { Mastrús; mapá; urucú; almécega; mamoeiro; são-caetano; erva de Santa Luzia; } \\
\text { carrapateiro; angico; alcaçús; copahiba; tamarindo; almiscar; sapucaia; erva de } \\
\text { passarinho; andirobeira; bútua; gameleira; maracujá; erva de bixo; contraerva; raiz } \\
\text { de cobra; cipó de cobra; quina; ipecacuanha; erva de xumbo; gengibre; ananozeiro. }\end{array}$ & 28 & 15 \\
\hline 4 & Utensílios & $\begin{array}{l}\text { Páo de remo; carnaúba; pindobeira; cuieira; buxa de paulista; sambahiba; sapucaia; } \\
\text { sumaúma; taboca; tocoára; cipó; coqueiro; sapucaia. }\end{array}$ & 13 & 7 \\
\hline 5 & Fibra & $\begin{array}{c}\text { Croatá; coqueiro; páo de envira; sapucaia; kiabeiro; pente de macaco; ananozeiro; } \\
\text { bananeira; tucum; tuari; algodoeiro }\end{array}$ & 11 & 6 \\
\hline 6 & Tóxica & $\begin{array}{l}\text { Mangabeira; sacáca; mandioca, macaxeira; urtiga de rato; páo de rato; } \\
\text { timbó; erva de rato; moconambi. }\end{array}$ & 9 & 5 \\
\hline 7 & Corante & Urucú; catinga de porco; anil; campéxe; gengibre; abacateiro & 6 & 3 \\
\hline 8 & Construção & Carnaúba; murutim; pindobeira; mangue branco; mangue vermelho; paparaúba. & 6 & 3 \\
\hline 9 & Óleo/Azeite & Urucurâna; carrapateiro; copahiba; andirobeira; anajá. & 5 & 3 \\
\hline 10 & Bebida & Erva de bixo; cafezeiro; gramo da terra; coqueiro; & 4 & 2 \\
\hline 11 & Saponáceas & São-caetano; saboneteiro; & 2 & 1 \\
\hline 12 & Fumo & Fumo ou tabaco & 1 & 0,5 \\
\hline 13 & Lenha & Candeia & 1 & 0,5 \\
\hline 14 & Incenso & Merim & 1 & 0,5 \\
\hline 15 & Ligante & Taquipé & 1 & 0,5 \\
\hline 16 & Cera & Urucurâna & 1 & 0,5 \\
\hline 17 & Aromática & Cumarú & 1 & 0,5 \\
\hline \multicolumn{3}{|r|}{ Total } & 189 & $\cong 100$ \\
\hline
\end{tabular}

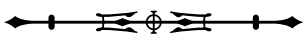


